\title{
Understanding operational regimes of closed loop pulsating heat pipes: an experimental study
}

\author{
Sameer Khandekar *, Nicolas Dollinger, Manfred Groll \\ Institut für Kernenergetik und Energiesysteme, Universität Stuttgart, 70550 Stuttgart, Germany
}

Received 14 August 2002; accepted 21 November 2002

\begin{abstract}
Increasing performance of electronic components is resulting in higher heat flux dissipation. Two-phase passive devices are proven solutions for modern microelectronics thermal management. In this context, heat pipe research is being continuously pursued evolving newer solutions to suit present requirements. Pulsating heat pipes (PHPs), a relatively new and emerging technology is one such field of investigation. The operating mechanism of PHP is not well understood and the present state of the art cannot predict required design parameters for a given task. The aim of research work presented in this paper is to better understand these mechanisms through experimental investigations.

Experiments were conducted on a PHP made of copper capillary tube of 2-mm inner diameter. Three different working fluids viz. water, ethanol and R-123 were employed. The PHP was tested in vertical (bottom heat mode) and horizontal orientation. The results strongly demonstrate the effect of input heat flux and volumetric filling ratio of the working fluid on the thermal performance of the device. Important insight into the operational regimes of the device has been gained.

(C) 2003 Elsevier Science Ltd. All rights reserved.
\end{abstract}

Keywords: Electronics cooling; Pulsating heat pipe; Single phase thermosyphon

\section{Introduction}

Meandering tube pulsating heat pipes, (PHPs), as proposed by Akachi et al. [1] have already found some applications in micro- and power electronics applications owing to favorable operational characteristics coupled with relatively cheaper costs. Although grouped as a subclass of

\footnotetext{
* Corresponding author. Tel.: +49-711-685-2142; fax: +49-711-685-2010.

E-mail address: khandekar@ike.uni-stuttgart.de (S. Khandekar).
} 


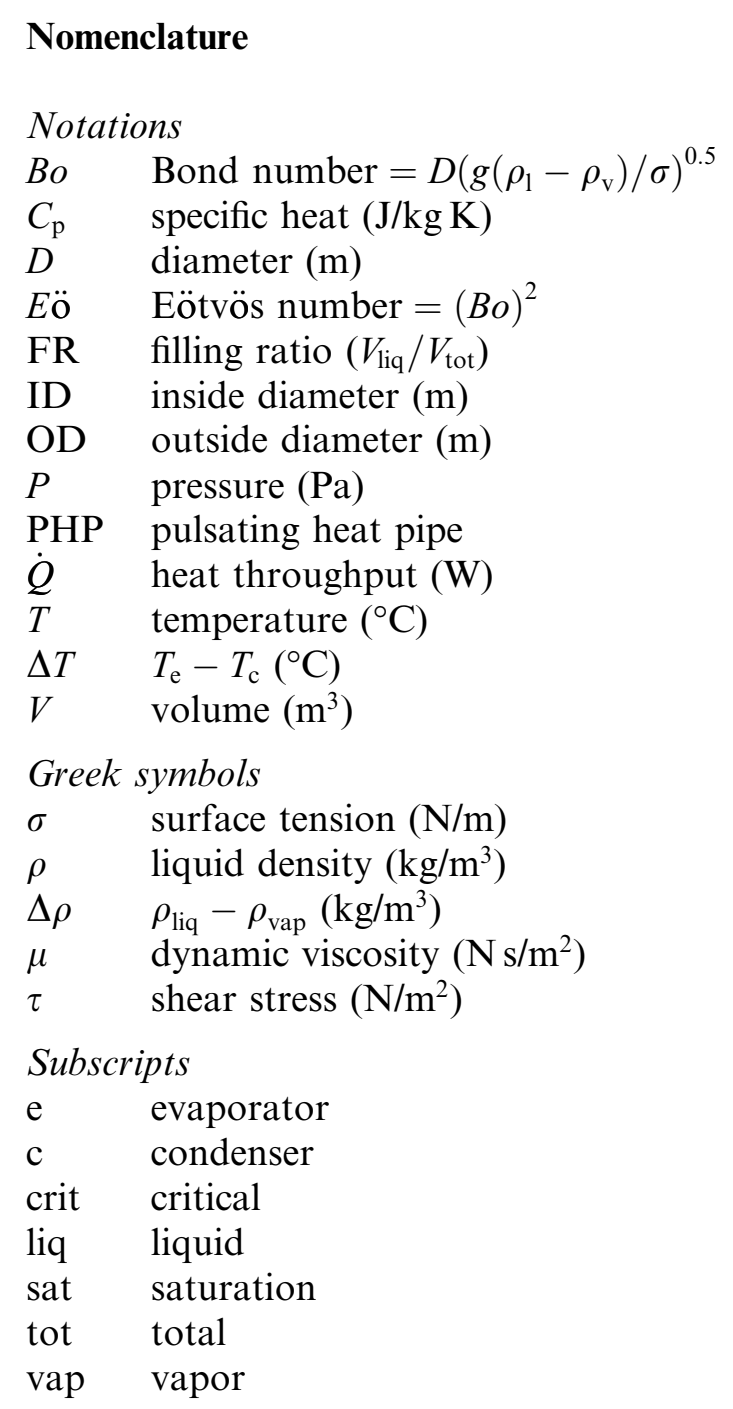

the overall family of heat pipes, the subtle complexity of thermofluidic transport phenomena is quite unique justifying the need of a completely different research outlook. Comprehensive theory of operation and reliable database or tools for the design of PHPs according to a given microelectronics-cooling requirement is still an unrealized task.

A closed loop pulsating or oscillating heat pipe consists of a metallic tube of capillary dimensions wound in a serpentine manner and joined end to end. It is first evacuated and then filled partially with a working fluid, which distributes itself naturally in the form of liquid-vapor slugs and bubbles inside the capillary tube. Respective tube sections thus have a different volumetric phase distribution. One end of this tube bundle receives heat transferring it to the other end by a pulsating action of the liquid-vapor slug-bubble system. There may exist an optional adiabatic 
zone in between. This type of heat pipe is essentially a non-equilibrium heat transfer device. The performance success primarily depends on continuous maintenance or sustenance of these nonequilibrium conditions in the system. The liquid and vapor slug/bubble transport is caused by the pressure pulsations inside the device. Since these pressure pulsations are fully thermally driven, because of the inherent construction of the device, there is no external mechanical power source required for the fluid transport.

In a working PHP, there exist temperature gradients between the evaporator and the condenser section. These are coupled with inherent real-time perturbations, due to:

- local non-uniform heating and cooling within the evaporator and condenser tube sections,

- unsymmetrical liquid-vapor distributions causing uneven void fraction in the tubes, and,

- presence of approximately triangular or saw-tooth alternating component of pressure drop superimposed on the average pressure gradient in a capillary slug flow due to the presence of vapor bubbles [2].

The net effect of all these temperature gradients and perturbations is to create a non-equilibrium pressure condition which, in conjunction with the non-uniform void fraction distribution in respective tubes, as stated earlier, is the primary driving force for thermofluidic transport. Thus self-sustained thermally driven oscillations are obtained $[3,4]$.

\section{Choice of working fluids}

The experience gained so far by earlier studies [4-8] suggests that the working fluid employed for PHPs should have the following properties:

- High value of $(\mathrm{d} P / \mathrm{d} T)_{\text {sat }}$ : ensuring that a small change in $T_{\mathrm{e}}$ generates a large corresponding $P_{\text {sat }}$ inside the generated bubble which aids in the bubble pumping action of the device. The same is true in reverse manner in the condenser.

- Low dynamic viscosity: which generates lower shear stress.

- Low latent heat: while prima facie, the two-phase flow characteristics of a PHP apparently suggest that latent heat may also play a sizable role in thermal performance, it is quite the contrary. The heat transfer is primarily due to sensible heat transport by the liquid [4,7] and therefore it may be argued that a low latent heat is in fact more beneficial, aiding quick bubble generation and collapse.

- High specific heat: is desirable, given the fact that sensible heat is playing the major role in heat transfer in the pulsating mode of PHP operation; although there are no specific studies which explicitly suggest the effect of specific heat of the liquid on the thermal performance. It is to be noted that if a flow regime change from slug to annular takes place the respective roles of latent and sensible heat transport mechanism may considerably change. The probability of annular flow is high with a combination of high Eötvös number, high heat flux and comparatively low FR $(\approx 50 \%$ or lower). This aspect requires further investigation.

- Low surface tension: which, in conjunction with dynamic contact angle hysteresis may create additional pressure drop [6]. 


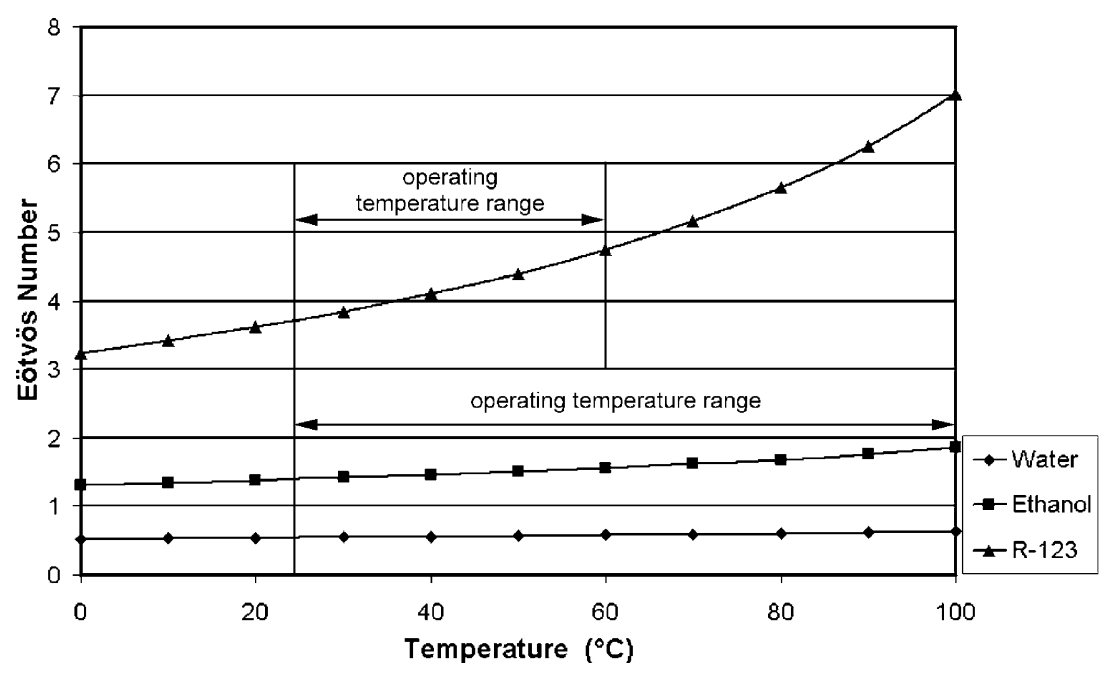

Fig. 1. Eötvös number variation of the working fluids in the operating temperature range.

From the construction of the device it is evident that distinct liquid slugs and vapor bubbles are essential for PHP operation. The formation of slugs in a capillary tube is attributed to the balance of gravity and surface tension forces, leading to the definition of Eötvös number [9] or alternatively, the Bond number. The theoretical maximum tolerable inner diameter of the PHP capillary tube is given as [1]: $D_{\text {crit }}=2\left[\sigma / g\left(\rho_{\text {liq }}-\rho_{\text {vap }}\right)\right]^{1 / 2}$ or $E \ddot{\mathrm{o}}=(B o)^{2}=4$. At diameters below this value there is a tendency of surface tension forces to predominate and this assists in formation of stable liquid slugs, an essential prerequisite for PHP operation. As the PHP tube diameter increases, the surface tension is reduced leading to stratification of phases. Therefore it seems to follow that above a maximum critical diameter the device will stop functioning as a PHP. The device may rather function as an interconnected array of two-phase thermosyphons.

For a better understanding of the effect of the above mentioned physical properties and the maximum critical diameter on the performance of the device, the present experiments were planned with water, ethanol and R-123. These three fluids have strongly different thermophysical properties, so that they can be looked as easily distinguishable representative working fluids. The variation of Eötvös number with temperature is given in Fig. 1. In the present series of experiments, while the Eö number for water and ethanol was well below the prescribed upper limit in the entire range of operating temperature $\left(\approx 25-100{ }^{\circ} \mathrm{C}\right)$, the $E$ ö number for R-123 crossed the limit of $E \ddot{o}=4$ in the operating range $\left(\approx 25-60^{\circ} \mathrm{C}\right)$.

\section{Experimental setups}

The schematic of the tested PHP is as shown in Fig. 2. The evaporator part of the PHP was made up of an aluminum block $\left(125 \times 30 \times 5 \mathrm{~mm}^{3}\right)$ heated by two electrical surface mounted and series connected resistance heaters of $5 \Omega$ resistance each. Thermal grease was applied to decrease thermal contact resistances. 


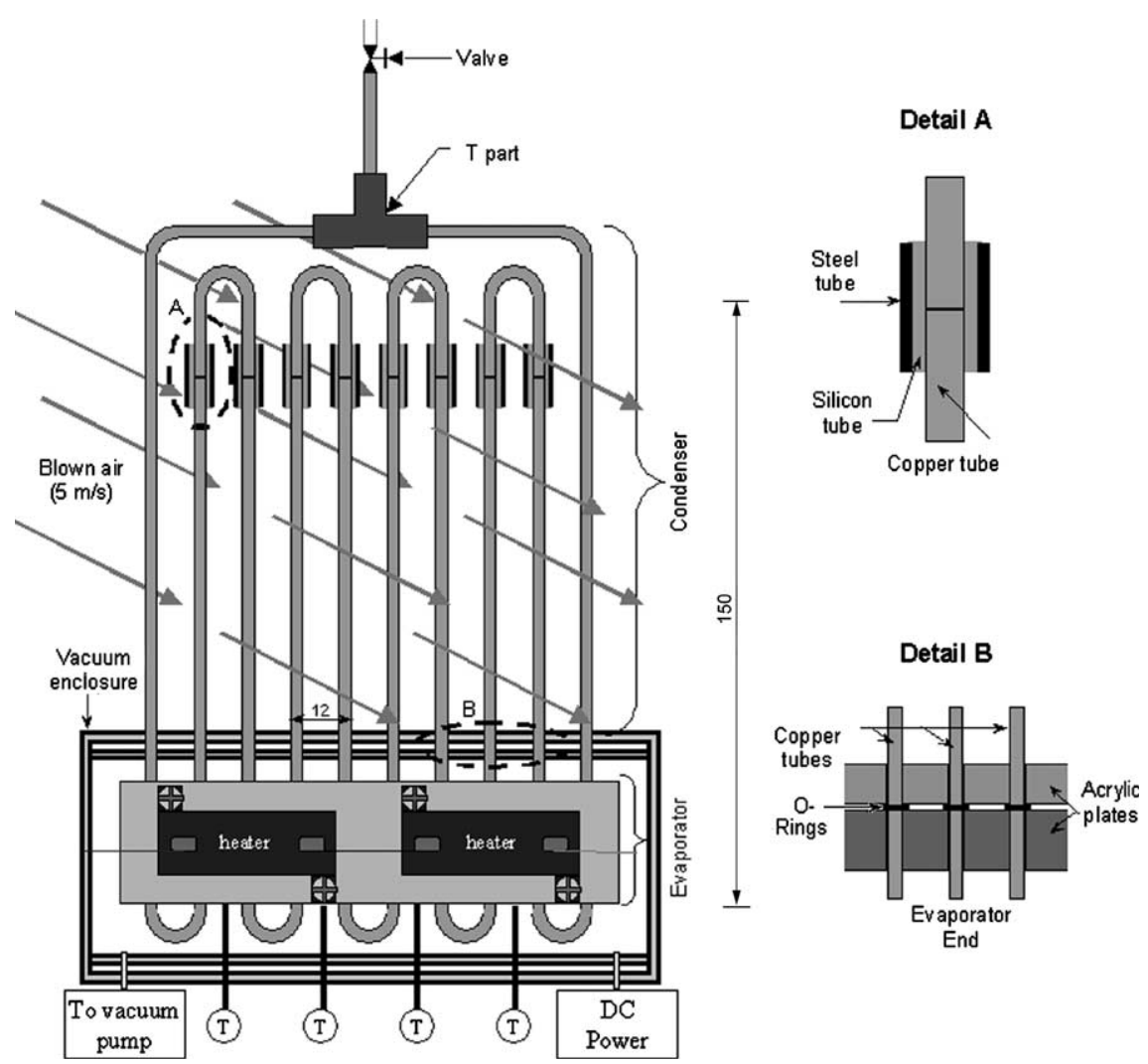

Fig. 2. Schematic of the experimental setup.

Copper tubes of ID $2 \mathrm{~mm}$ and OD $3 \mathrm{~mm}$ were bent in a U-shape. Two of the bends located at two extreme ends were kept longer on one side in order to complete the closed loop circuit through the T-connector. Four small U-turns were also made from the same pipe forming the junctions with the main tubes as shown. Before connecting the copper U-tubes together, they were inserted in suitably sized and located holes in the aluminum evaporator block. A mechanical interference fit was ensured to give an excellent thermal contact.

The final connection between these copper U-turns was made with flexible silicon tubes. An additional small steel tube was also fixed as a sleeve on the silicon tube connecting the copper bends to resist the likely deformation of the silicon tubes in case of over-pressure. The T-connector was fitted with a filling/metering valve. The final PHP assembly was tested under vacuum and a pressure of $10^{-4}$ mbar could be easily maintained.

Air-cooling was applied by blowing air directly on the bare copper tubes by a centrifugal blower and connecting wind tunnel arrangement at average air velocity of $5 \mathrm{~m} / \mathrm{s}$ and temperatures ranging from 24 to $28{ }^{\circ} \mathrm{C}$.

For achieving good insulation, the evaporator assembly was fully enclosed in an acrylic glass box inside which a low pressure $\left(<10^{-3}\right.$ mbar $)$ was maintained by continuously running a vacuum pump. A re-radiating foil shield was placed around the evaporator to prevent radiation losses. The PHP copper tubes passed out of the acrylic box through 10 holes with O-ring seals ensuring 
leak tightness. The entire setup along with the PHP, wind tunnel and blower could be turned to a desired inclination angle.

For temperature measurements, a data logger (resolution $0.1{ }^{\circ} \mathrm{C}$, accuracy $\pm 0.5{ }^{\circ} \mathrm{C}$, time constant $70 \mathrm{~ms}$ ) was employed with a measuring frequency of $1 \mathrm{~Hz}$, coupled with ungrounded $\mathrm{K}$ type thermocouples (Thermacoax) of $1.5 \mathrm{~mm}$ diameter. A worst case analysis indicates the accuracy of measurement for maximum heat throughput was below $9 \%$. The results given in Section 5 for the heat throughput are averaged-out indicative values of a series of repeated tests. A total of six thermocouples were used in the setup. Two thermocouples were located in the wind tunnel path measuring the average condensing air temperature (average $T_{\mathrm{c}}$ ). The other four thermocouples were located in the drilled passages in the aluminum evaporator block. These four thermocouples passed through the insulating vacuum box around the evaporator. O-rings around them ensured the vacuum tightness of the box. The thermocouples were inline with the copper PHP tubes but were located at different positions inside the aluminum plate so as to give an average representative value of the aluminum block temperature (average $T_{\mathrm{e}}$ ). All these thermocouples were connected to a PC via the data logger. A precise DC power supply (resolution 0.01 $\mathrm{V} / 0.01 \mathrm{~A})$ provided the necessary power input.

\section{Experimental procedure}

For filling the working fluid into the PHP, one of the connector flexible silicon tubes in the condenser was removed and the working fluid was filled using a syringe injector. The silicon tube was put back onto the copper tube again always ensuring that there was no air bubble left in the tube. In this way the PHP volume was filled with $100 \%$ working fluid. This filling ratio was then reduced to the desired value by sucking through the metering valve, which was fitted to a vacuum pump. Since the weight of the PHP in the dry state and in the $100 \%$ filled state was previously measured, the exact filling ratio could be calculated.

At fixed filling ratio, input heat power was varied till the average evaporator temperature reached $100{ }^{\circ} \mathrm{C}$ (in case of water and ethanol) or $60{ }^{\circ} \mathrm{C}$ (in case of R-123). Since the saturation pressure of $\mathrm{R}-123$ at $100{ }^{\circ} \mathrm{C}$ (about 7.8 bars) is too high for safe operation of the copper PHP due to the presence of flexible silicon tubing, all experiments with R-123 were carried out till a maximum $T_{\mathrm{e}}=60{ }^{\circ} \mathrm{C}$. It is clear from Table 1 that even at this low operating temperature (i.e. $T_{\mathrm{e}}=60^{\circ} \mathrm{C}$ ), R-123 has a much better pressure rise response than ethanol and water. The thermal resistance of the PHP, in all cases, was evaluated based on the steady state average evaporator temperature and the corresponding condensing air temperature. Since the evaporator was completely vacuum insulated, the thermal power was directly calculated from the source electrical

Table 1

Comparison of thermophysical properties of the working fluids

\begin{tabular}{|c|c|c|c|c|c|c|c|}
\hline \multirow[t]{2}{*}{ Fluid } & \multicolumn{2}{|l|}{$P_{\text {sat }}$ (bar) } & \multicolumn{2}{|l|}{$\rho_{\text {liq }}\left(\mathrm{kg} / \mathrm{m}^{3}\right)$} & \multirow{2}{*}{$\begin{array}{l}\mu_{\text {liq }}\left(\mathrm{N} \mathrm{s} / \mathrm{m}^{2}\right) \\
\text { at }\left(T_{\mathrm{c}}+T_{\mathrm{e}}\right) / 2\end{array}$} & \multirow{2}{*}{$\begin{array}{l}C_{\mathrm{p}-\text { liq }}(\mathrm{J} / \mathrm{kg} \mathrm{K}) \\
\text { at }\left(T_{\mathrm{c}}+T_{\mathrm{e}}\right) / 2\end{array}$} & \multirow[t]{2}{*}{$\mathrm{d} P /\left.\mathrm{d} T\right|_{\text {sat }}(\operatorname{bar} / \mathrm{K})$} \\
\hline & at $T_{\mathrm{c}}$ & at $T_{\mathrm{e}}$ & at $T_{\mathrm{c}}$ & at $T_{\mathrm{e}}$ & & & \\
\hline Water & 0.0317 at $25^{\circ} \mathrm{C}$ & 1.0132 at $100^{\circ} \mathrm{C}$ & 997 at $25^{\circ} \mathrm{C}$ & 958 at $100{ }^{\circ} \mathrm{C}$ & $453.2 \mathrm{e}-6$ & $4.183 \mathrm{e} 3$ & 0.013 \\
\hline Ethanol & 0.0787 at $25^{\circ} \mathrm{C}$ & 2.255 at $100^{\circ} \mathrm{C}$ & 785 at $25^{\circ} \mathrm{C}$ & 714 at $100{ }^{\circ} \mathrm{C}$ & $560.8 \mathrm{e}-6$ & $2.805 \mathrm{e} 3$ & 0.029 \\
\hline $\mathrm{R}-123$ & 0.913 at $25^{\circ} \mathrm{C}$ & 2.862 at $60{ }^{\circ} \mathrm{C}$ & 1463 at $25^{\circ} \mathrm{C}$ & 1370 at $60{ }^{\circ} \mathrm{C}$ & $350.7 \mathrm{e}-6$ & $1.047 \mathrm{e} 3$ & 0.056 \\
\hline
\end{tabular}


power. After reaching a steady state in vertical orientation the setup was immediately turned to the horizontal mode and allowed to achieve a quasi-steady state at the new orientation. Suitable corrections were applied after accounting for the losses.

\section{Experimental results}

Fig. 3 shows the maximum heat throughput achievable in vertical bottom heat mode for reaching the average evaporator temperature of $100^{\circ} \mathrm{C}$ (for water and ethanol) and $60{ }^{\circ} \mathrm{C}$ (for R123). Figs. 4-6 show the variation of overall thermal resistance $\left(=\left(T_{\mathrm{e}}-T_{\mathrm{c}}\right) / \dot{Q}\right)$ of the PHP with increasing heat input power $\dot{Q}$ for different filling ratios for water, ethanol and R-123 for vertical bottom heat mode operation.

\subsection{Operational extremities}

For $0 \%$ filling ratio, the PHP runs without any fluid inside it and this serves as the reference measurement. Obviously the mode of heat transfer is by pure conduction through the copper material and not more than about $21 \mathrm{~W}$ of heat could be transported to achieve an average evaporator temperature of $100{ }^{\circ} \mathrm{C}$ at a thermal resistance of $3.6 \mathrm{~K} / \mathrm{W}$.

The other extreme is when the PHP is fully filled with the working fluid $(\mathrm{FR}=100 \%)$. In this mode the heat transfer is due to the single-phase buoyancy induced liquid circulation in the PHP (this fact has also been confirmed by seeding the flow with tracer particles and the observation of alternating hot and cold PHP tubes in earlier experiments with a glass tube PHP reported in [10]). In this case the local heat transfer coefficient in the tubes is only a function of fluid Grashof and Prandtl numbers. There is a smooth decrease of the thermal resistance with increasing heat power input. A corresponding typical time-temperature transient response of the average evaporator

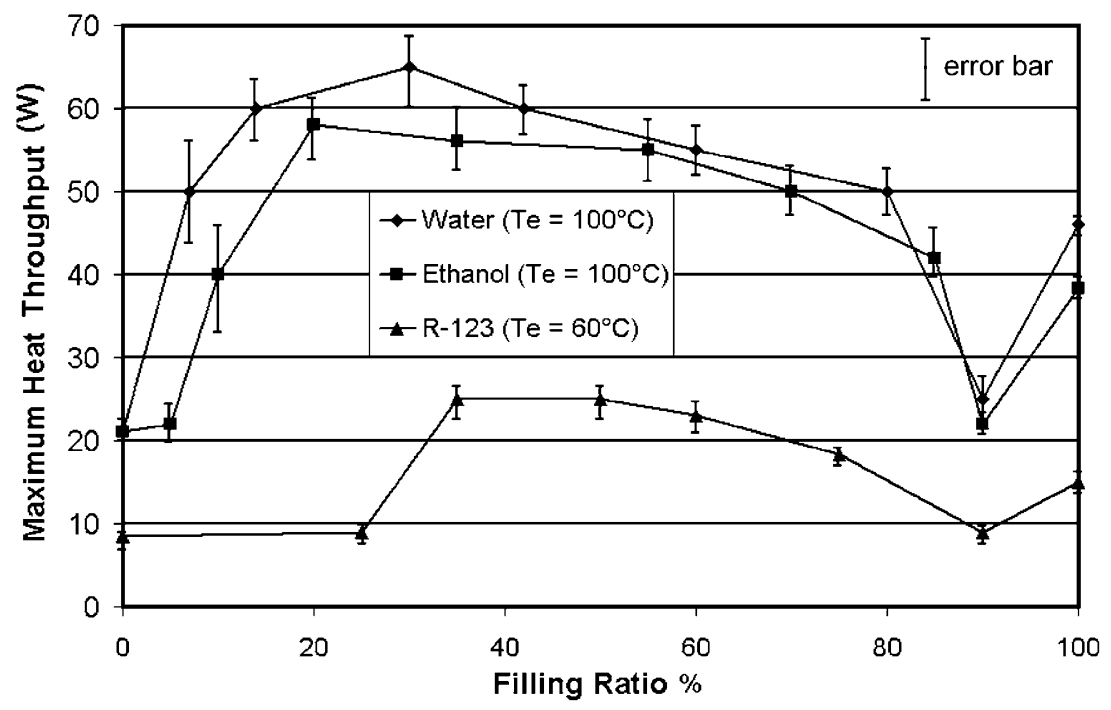

Fig. 3. Maximum performance of PHP obtained with respect to the filling ratio. 


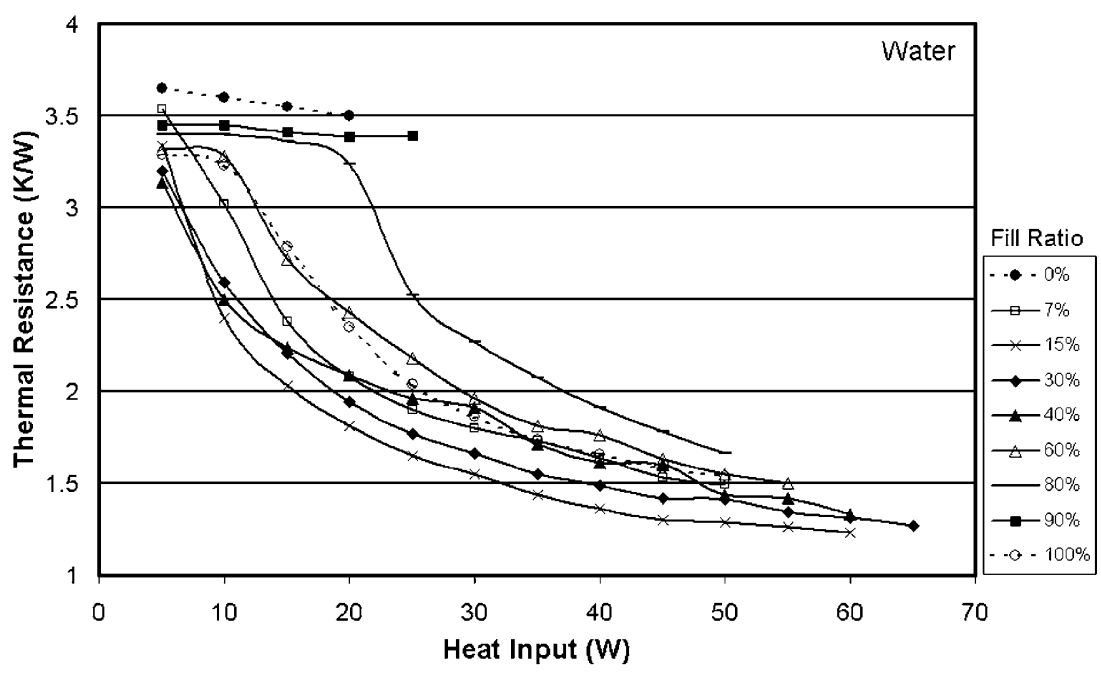

Fig. 4. Typical variation of thermal resistance with heat input power at different fill ratios for water filled PHP.

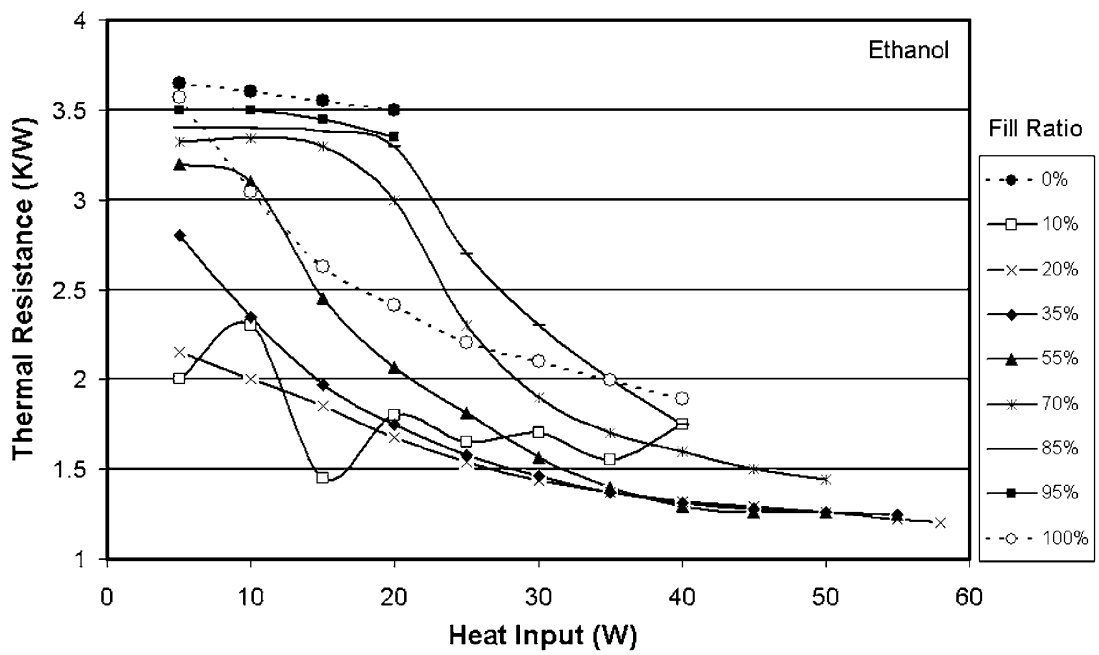

Fig. 5. Typical variation of thermal resistance with heat input power at different fill ratios for ethanol filled PHP.

temperature for water is shown in Fig. 7(a). With increasing heat input to the device, the evaporator temperature rises resulting in a greater density gradient in the tubes. Simultaneously the liquid viscosity also drops diminishing the wall friction. For power input of 5 and $10 \mathrm{~W}$, the circulation is not initiated. At $15 \mathrm{~W}$, there is a sudden decrease of $T_{\mathrm{e}}$ as the liquid circulation starts. The respective adjacent tubes of the PHP become and remain alternatively hot and cold thereafter. The circulation direction which the liquid takes is arbitrary, remaining fixed for a given experiment but may change with different experimental runs. The circulating liquid velocity keeps rising thereafter resulting in a thermal response as depicted. 


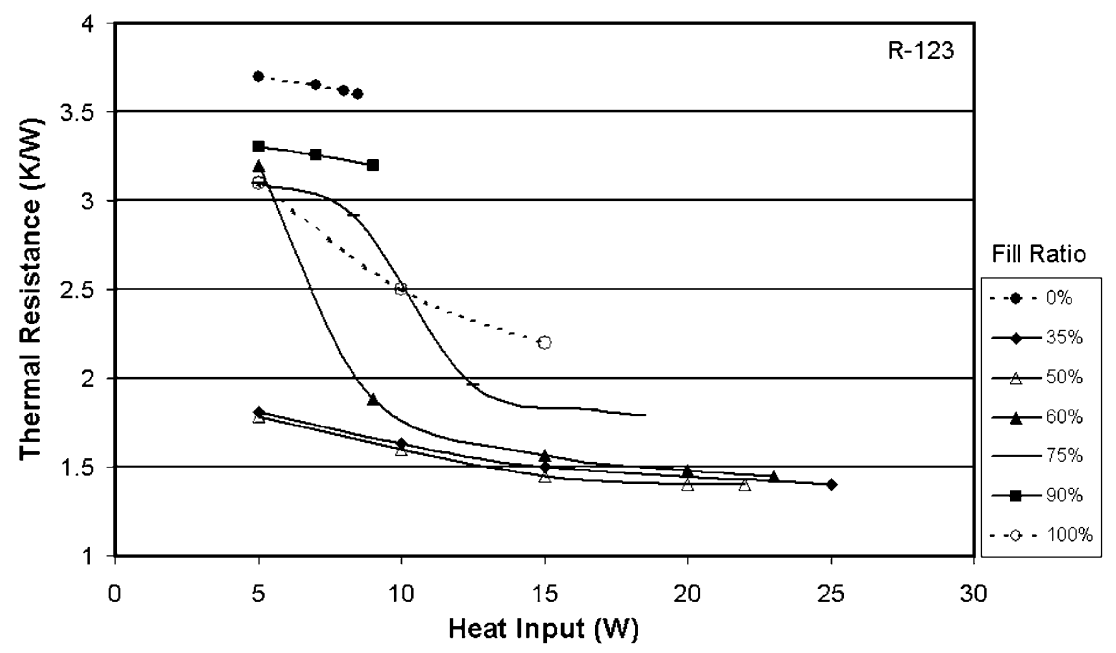

Fig. 6. Typical variation of thermal resistance with heat input power at different fill ratios for R-123 filled PHP.
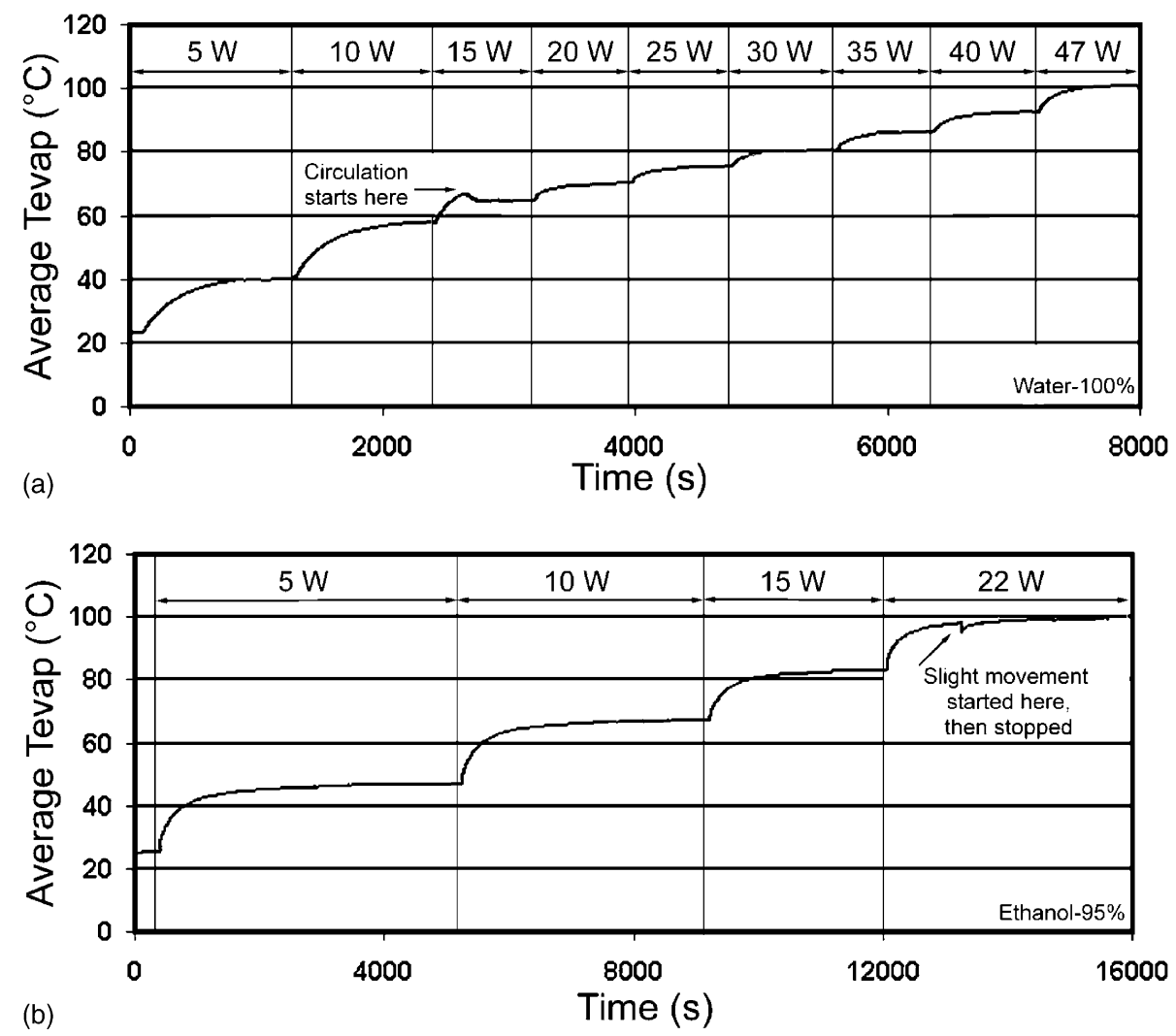

Fig. 7. (a) Transient variation of $T_{\mathrm{e}}$ for $\mathrm{FR}=100 \%$ and water as the working fluid, (b) $\mathrm{FR}=95 \%$ and ethanol as the working fluid. 
A dry PHP on the one hand and a fully filled PHP on the other serve as the reference for the operation of the device.

\subsection{Pulsating mode of operation}

\subsubsection{Vertical, bottom heating operation mode}

As soon as a very small amount of the working fluid is sucked out of the device ( $\approx 95 \%$ fill $)$, it results in the formation of few bubbles in the tubes. There is a remarkable drop in performance with the introduction of these first few bubbles. In the single-phase mode $(100 \%$ fill $)$, the liquid could freely circulate in the tube resulting in substantial convective heat transfer. As soon as there is a single bubble in the device, this naturally circulating flow is hindered. Under the new working conditions, the driving force generated due to the density gradient has to overcome additional forces to induce a flow. These new retarding forces are due to (a) the additional frictional resistance (or pressure drop) created due to the head and tail section of the bubble, (b) the buoyancy force which acts on the bubble due to which it is difficult to bring the bubble in the downward direction against the gravity. Also, since nearly $95 \%$ of the PHP is filled with an incompressible fluid, the overall degree of freedom is very limited. A closer look at the time-temperature curve for ethanol (Fig. 7(b)) at this filling ratio shows that at $22 \mathrm{~W}$ heat power input a slight momentary depression in the evaporator temperature is observed. In such a situation it has been observed that bubbles temporarily move a small distance (i.e. there is an ephemeral circulation) but again get stationary after a while [10]. The liquid density gradient cannot not initiate any permanent flow in the device.

Between about $20 \%$ and $70 \%$ filling ratio, depending on the working fluid, the device functions in a true pulsating mode. The thermal performance improves, until a lower minimum fill charge, below which intermittent operation with partial or total dryout in the evaporator section commences. For water, the maximum performance was observed at about 15-30\% filling ratio. For ethanol and R-123, a range of filling ratios between 25\% and 55\% (ethanol) and 35-60\% (R-123) gave maximum heat throughput.

A partially filled PHP operated better than a 100\% filled (single-phase thermosyphon mode) device only under certain operating conditions. For example, at $85 \%$ filling ratio for ethanol, the maximum thermal power transported was about $42 \mathrm{~W}$ to attain an average evaporator temperature of $100{ }^{\circ} \mathrm{C}$. At this power it was only a slight improvement over the $100 \%$ filled mode. For all the heating power range below $42 \mathrm{~W}$, the performance of the $85 \%$ filled PHP was inferior to that of the $100 \%$ filled PHP (see Fig. 5). Up to $20 \mathrm{~W}$ there was hardly any pulsating effect inside the tubes. Only after crossing this limit, macrolevel movements initiated resulting in improved performance. At lower heating powers $T_{\mathrm{e}}$ and corresponding $P_{\text {sat }}$ is quite low. The overall degree of freedom of the device is also still quite low due to the presence of only a small number of bubbles. Simultaneously the presence of bubbles creates additional frictional pressure drop and the advantage likely to be achieved due to their presence is not really observed under such working conditions. Below filling ratio of $55 \%$ and up to $20 \%$, there is a clear advantage of a PHP as compared to a $100 \%$ filled mode of operation. In this case, the pulsating action inside the PHP gets initiated earlier at lower heat inputs.

Typical transient response of a ethanol, 55\% filled PHP is shown in Fig. 8(a). Practically no pulsations are initiated till $15 \mathrm{~W}$ heat input. Thereafter the device functions better than in the $100 \%$ filled mode of operation and self-sustained thermally driven oscillations are continuously 

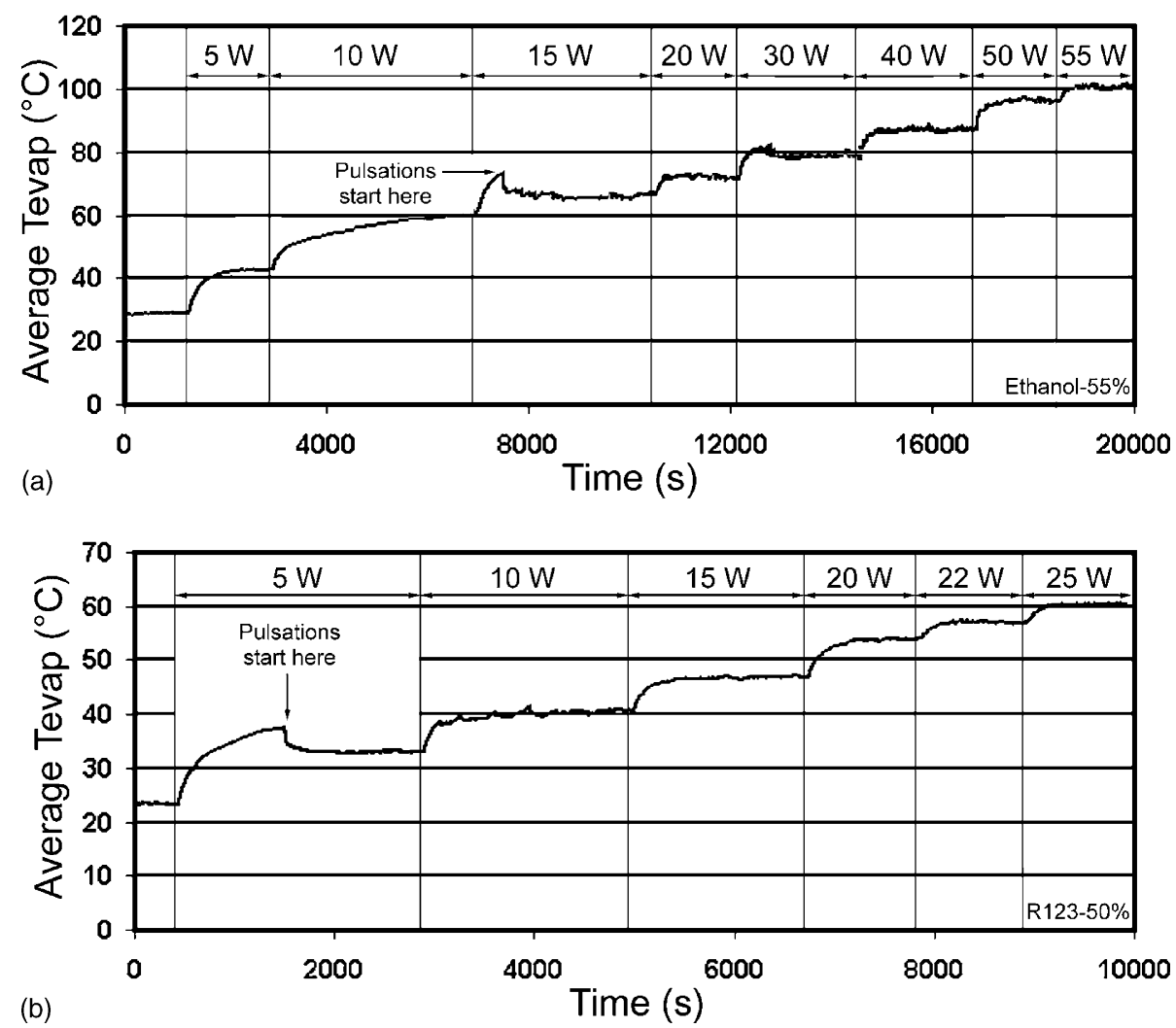

Fig. 8. (a) Transient variation of $T_{\mathrm{e}}$ for $\mathrm{FR}=55 \%$ and ethanol as the working fluid, (b) $\mathrm{FR}=50 \%$ and $\mathrm{R}-123$ as the working fluid.

maintained. Similar results for R-123 (50\% filled) are shown in Fig. 7(b) where the pulsations initiate at a low power input of $5 \mathrm{~W}$ only. At this filling ratio of R-123, the PHP is better than the $100 \%$ filled device at all power ranges as seen in Fig. 6.

In vertical bottom heat mode the vapor bubbles which take up heat in the evaporator grow in size. Their own buoyancy helps them to rise up in the tube section. Simultaneously other bubbles, which are above in the tube, are also helped by their respective buoyant forces. These rising bubbles in the tube also have to carry the liquid slugs trapped in between them. In this mode of operation there is a natural tendency for the liquid slugs to travel downwards, helped by gravity force, towards the evaporator. Simultaneously the vapor bubbles have the natural tendency to travel towards the condenser helped by their buoyancy.

\subsubsection{Horizontal operation mode}

In this mode of operation the PHP did not work as expected with any of the working fluids. In fact there was hardly any macromovement of bubbles and the thermal resistance was practically constant and comparable to the dry PHP $(0 \%$ filled $)$. This strongly suggests that although the Eötvös number of ethanol and water is well below the suggested critical value (see Fig. 1), gravity does play a role in the PHP action (at least at this diameter for ethanol). 
Since gravity force is absent, all the movement of bubbles and slugs has to be necessarily done by the pressure forces. These forces are created due to temperature differences, which exist between evaporator and condenser. The uneven heating and cooling which always exists in real systems further contributes by creating additional perturbations. In the present series of experiments, the pulsating action in the horizontal orientation could not be initiated at all. The performance, in this case, was comparable to the dry ( $\% \%$ filled) device. This strongly suggests that the necessary pressure forces coupled with perturbations were not strong enough for creating pulsations. This situation may be improved by the following:

If the same fluid is to be used, the PHP should operate at a higher $T_{\mathrm{e}}$, keeping the $T_{\mathrm{c}}$ constant. This will raise the driving pressure difference in the device. If the same $T_{\mathrm{e}}$ is to be maintained then some other working fluid having higher $P_{\text {sat }}$ at this temperature as well as a steeper $(\mathrm{d} P / \mathrm{d} T)_{\text {sat }}$ should be used. In general, it is also expected that an increase in number of turns of the device should increase the level of perturbations. This might be a possible solution for better operation in the horizontal mode.

\section{Conclusions}

The following are the main conclusions of the study:

1. Valuable information related to the fundamental characteristics and operational regimes of a PHP were generated. For a given heat throughput requirement, an operationally better performing and self-sustained thermally driven pulsating action of the device was only observed in the filling ratio range $25-65 \%$ depending on the working fluid. Above this range, the overall degree of freedom and the pumping action of bubbles was insufficient for rendering good performance. Below a certain range of filling ratio, partial dryout of the evaporator was detected.

2. The results also indicate that a 100\% filled PHP (not working in the pulsating mode but instead as a single-phase buoyancy-induced thermosyphon) is thermally better performing than a partially filled pulsating mode device under certain operating conditions.

3. The tested PHP did not operate in the horizontal mode for all the working fluids tested. The reasons are attributed to small number of turns and too low operating pressures existing at testing conditions.

4. Although the Eötvös number of water and ethanol was much below the prescribed maximum limit of $E \ddot{o}=4$, gravity forces were definitely seen to affect the performance. Similarly, while the $E \ddot{o}$ number of R-123 is higher than the maximum suggested limit for the working temperature range, the PHP still worked quite effectively in the vertical mode. This suggests that though at $E \ddot{O}>4$, while the tendency of slug flow diminishes as surface tension tends to reduce, a certain amount of fluid transport is still possible by the bubble pumping action thereby providing substantial heat transfer.

\section{Acknowledgements}

This research has been partly supported by Deutsche Forschungsgemeinschaft (DFG) under grant number GR 412/33-1. 


\section{References}

[1] H. Akachi, F. Polášek, P. Štulc, Pulsating heat pipes, Proceedings of the 5th International Heat Pipe Symposium, Melbourne, Australia, 1996, 208-217 (ISBN 0-08-042842-8).

[2] G. Wallis, One Dimensional Two-Phase Flow, McGraw Hill Inc., 1969 (ISBN 0-0706-794-28).

[3] S. Khandekar, M. Schneider, M. Groll, Mathematical modeling of pulsating heat pipes: state-of-the-art and future challenges, 5th ASME/ISHMT joint International Heat and Mass Transfer Conference, Kolkata, India, 2002, 856862 (ISBN 0-07-047443-5).

[4] M. Groll, S. Khandekar, Pulsating heat pipes: a challenge and still unsolved problem in heat pipe science, Proceedings of the 3rd International Conference on Transport Phenomena in Multiphase Systems, Kielce, Poland, 2002, 35-44 (ISBN 83-88906-03-8).

[5] S. Duminy, Experimental investigation of pulsating heat pipes, Diploma thesis, Institute of Nuclear Engineering and Energy Systems (IKE), University of Stuttgart, Germany, 1998.

[6] S. Khandekar, M. Schneider, P. Schäfer, R. Kulenovic, M. Groll, Thermofluid dynamic study of flat plate closed loop pulsating heat pipes, Microsc. Thermophys. Eng. 6 (4) (2002) 303-318 (ISSN 1089-3954).

[7] M.B. Shafii, A. Faghri, Y. Zhang, Thermal modeling of unlooped and looped pulsating heat pipes, ASME J. Heat Transfer 123 (2001) 1159-1172.

[8] B.Y. Tong, T.N. Wong, K.T. Ooi, Closed loop pulsating heat pipe, Appl. Therm. Eng. 21 (2001) 1845-1862 (ISSN 1359-4311).

[9] T.Z. Harmathy, Velocity of large drops and bubbles in media of infinite or restricted extent, AIChE 6 (2) (1960) 281-288.

[10] S. Khandekar, M. Groll, P. Charoensawan, P. Terdtoon, Pulsating heat pipes: thermofluidic characteristics and comparative study with single phase thermosyphon, 12th International Heat Transfer Conference, Grenoble, France, 2002, 459-464 (ISBN 2-84299-307-1). 